\title{
Vertical Electron Transport through PbS-EuS Structures
}

\author{
S. Wrotek ${ }^{a}, \mathrm{~K} . \mathrm{DyBkO}^{a}$, A. Morawski ${ }^{a}, \mathrm{~A} \mathrm{MAqKOSA}^{a}$, \\ T. Wosiński $^{a}$, T. Figielski ${ }^{a}, Z$ Z. TKACZYK ${ }^{a}$, E. EUSAKOWSKA $^{a}$ \\ T. Story ${ }^{a}$, A.Yu. Sipatov ${ }^{b}$ A. Szczerbakow ${ }^{a}$, K. Grasza ${ }^{a}$, \\ J. WróBel ${ }^{a}$ AND W. PALOSZ ${ }^{c}$ \\ ${ }^{a}$ Institute of Physics, Polish Academy of Sciences \\ al. Lotników 32/46, 02-668 Warsaw, Poland \\ ${ }^{b}$ National Technical University "KPI", Frunze Str. 21, 61002 Kharkov, Ukraine \\ ${ }^{c}$ US-NASA/Marshal Space Flight Center, Huntsville, Alabama 35812, USA
}

Temperature dependence of current-voltage $I-V$ characteristics and resistivity is studied in ferromagnetic PbS-EuS semiconductor tunnel structures grown on $n$-PbS (100) substrates. For the structures with a single (2-4 nm thick) ferromagnetic EuS electron barrier we observe strongly non-linear $I-V$ characteristics with an effective tunneling barrier height of $0.3-0.7 \mathrm{eV}$. The experimentally observed non-monotonic temperature dependence of the (normal to the plane of the structure) electrical resistance of these structures is discussed in terms of the electron tunneling mechanism taking into account the temperature dependent shift of the band offsets at the EuS-PbS heterointerface as well as the exchange splitting of the electronic states at the bottom of the conduction band of EuS.

PACS numbers: $75.20 . \mathrm{Ck}, 75.30 . \mathrm{Et}$

\section{Introduction}

EuS-PbS multilayers are all-semiconductor magnetic quantum structures in which ferromagnetic insulating layers of EuS are electronic barriers whereas non-magnetic conducting layers of PbS are quantum wells. Both these semiconductor compounds crystallize in the rock salt structure with well matching lattice parameters, $\left(a_{\mathrm{EuS}}-a_{\mathrm{PbS}}\right) / a_{\mathrm{PbS}}=0.5 \%$ at room temperature. It permits epitaxial growth of high crystal quality superlattices and other layered structures employing 
high vacuum deposition techniques [1]. Ferromagnetic transition in EuS-PbS multilayers is observed even in structures with ultrathin layers of EuS of only $0.6 \mathrm{~nm}$ (i.e., just two monolayers). The transition temperature of EuS-PbS multilayers decreases (from the value of $16.5 \mathrm{~K}$ observed in bulk crystals $[2,3]$ ) with decreasing thickness of EuS layer, and also depends on the strain brought about by the difference in the thermal expansion coefficients of $\mathrm{KCl}(100)$ and $\mathrm{BaF}_{2}$ (111) substrates and the multilayer $[4,5]$. The photoluminescence studies of EuS-PbS heterostructures showed that these heterostructures exhibit type-I ordering of conduction and valence band edges with narrow gap ( $0.3 \mathrm{eV}$ at low temperatures) $\mathrm{PbS}$ layers forming quantum wells for both holes and electrons [1, 6]. EuS-PbS multilayers constitute an intriguing new spintronic system in which one expects to control the electrical and optical properties of the structure by changing the magnitude and orientation of magnetization vectors of EuS barriers. The key physical effect here is the large spontaneous exchange splitting of the conduction band of EuS observed below the Curie temperature $[2,4]$. It results in a large temperature and magnetic field controlled change of the height of the electronic barrier for electrons at the EuS-PbS heterointerface. These effects were already observed in metal-EuS hybrid structures exhibiting very large tunneling magnetoresistance [7, 8]. In this respect, particularly interesting are EuS-PbS multilayers grown on $\mathrm{KCl}(100)$ and PbS (100) substrates in which, for very thin PbS spacer layers, the antiferromagnetic interlayer coupling is observed in neutron diffraction and magnetization experiments $[5,9,10]$. The ferromagnetic mutual orientation of magnetization vectors of EuS layers can be obtained by the application of small external magnetic field of the order of $10 \mathrm{mT}$ thus providing an efficient way of control of the magnetic state of the multilayer.

In all-semiconductor EuS-PbS structures, the search for new spintronic transport and optical effects brought about by the ferromagnetic character of electronic barriers is in its initial stage. The recent report showed, in particular, that the structures with double EuS ferromagnetic barrier exhibit strongly nonlinear $I-V$ characteristics with a range of voltages corresponding to negative differential resistance [11]. In this work, we report experimental studies of vertical (tunneling) electron transport through a model PbS-EuS-PbS heterostructures containing a single EuS ferromagnetic barrier with the nominal thickness of $2-4 \mathrm{~nm}$. We exploit here the technological approach to the vertical transport, developed in our Institute, which is based on the use of conducting $n$-PbS substrates [12-14].

\section{Growth and electrical measurements}

PbS-EuS(t)-PbS heterostructures with EuS thickness in the range $t=2-4 \mathrm{~nm}$ were epitaxially grown by high vacuum thermal evaporation of $\mathrm{PbS}$ and electron-beam evaporation of EuS on freshly cleaved (100)-oriented substrates of $n$-type PbS crystals. The substrate temperature during growth was about 


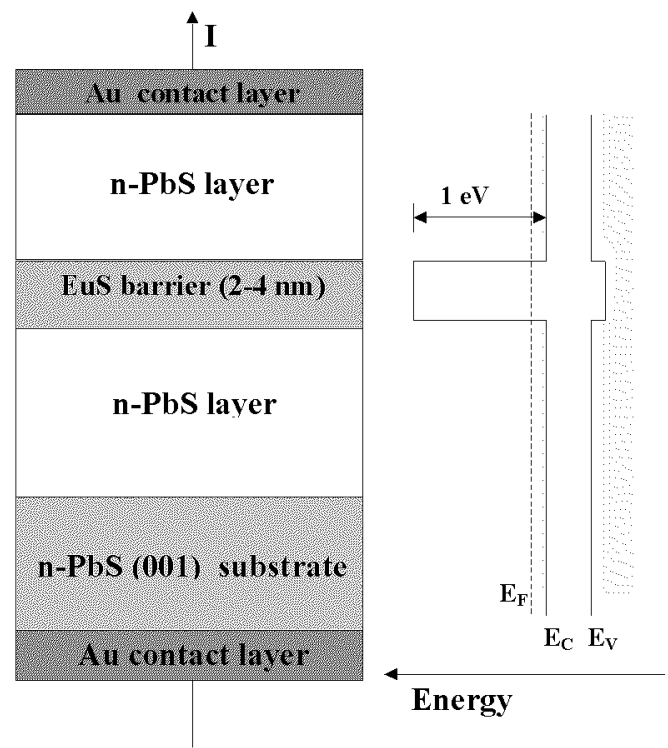

Fig. 1. A scheme of EuS-PbS multilayer structure with a single ferromagnetic barrier which is employed in this work to study the vertical electron transport. The bottom electrode is provided by conducting $n$ - $\mathrm{PbS}$ monocrystalline substrate with deposited layer of Au. The right hand side of the figure presents a model of energetic position of the edges of valence and conduction bands in EuS-PbS heterostructure along the growth direction of the multilayer.

$350^{\circ} \mathrm{C}$. The $\mathrm{PbS}$ monocrystals were grown in our Institute by various modifications of physical vapor transport method $[13,14]$. X-ray and atomic force microscopy (AFM) characterization of the crystal quality of a series of PbS substrate plates showed the X-ray rocking curve for the (200) reflex with the full width at half maximum (FWHM) parameter in the range 65-250 arcsec and the root-mean-square (rms) roughness of $1 \mathrm{~nm}$ for the analyzed area of $10 \times 10 \mu \mathrm{m}^{2}$. The characteristic feature of surface morphology of $\mathrm{PbS}$ substrate plates is the presence of long, practically atomically flat regions with typical dimensions of $(1-10) \times 0.1 \mu \mathrm{m}^{2}$. The FWHM rocking curve width for EuS-PbS structures grown on PbS substrates is typically 200-300 arcsec, whereas the AFM analysis (performed in air) of the morphology of the top PbS layer in the EuS-PbS multilayer showed rms roughness of $4 \mathrm{~nm}$ for the analyzed region of $10 \times 10 \mu \mathrm{m}^{2}$ [12]. The investigated samples in the form of rectangular pillars with the side dimensions $200-500 \mu \mathrm{m}$ were prepared by a direct cleavage of the heterostructures along the (010) and (001) crystal planes. The $n$-PbS substrate also served as a bottom electrode. The structures were capped with $100 \mathrm{~nm}$ PbS layer (see Fig. 1). The top electrical contact was made to this cap layer using either $\mathrm{Au}$ or $\mathrm{LaB}_{6}$ metallization and conducting carbon or silver pastes. 
We applied the standard dc method to measure the current, $I$, passing vertically (see Fig. 1) through the structure as a function of the applied voltage, $V$, in the temperature range $T=4.2-230 \mathrm{~K}$. The temperature dependence of resistance was studied at low dc bias $(V<100 \mathrm{mV})$ without external magnetic
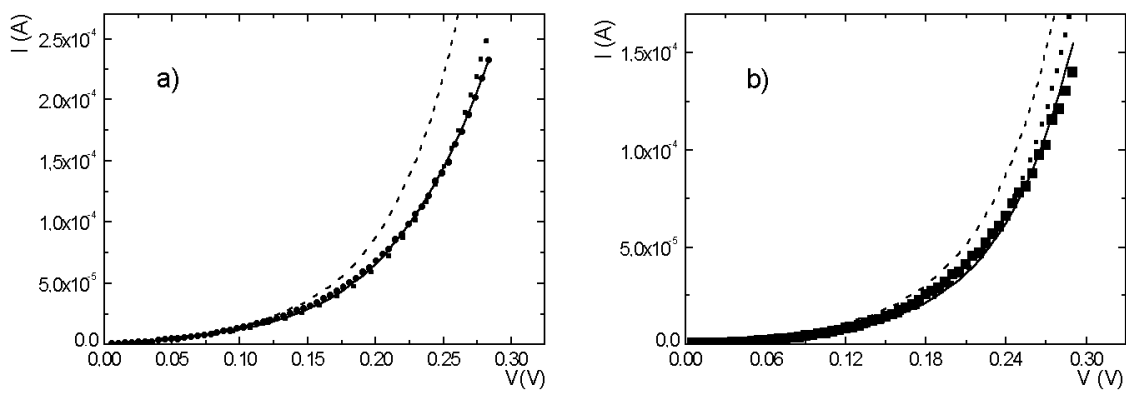

Fig. 2. The current-voltage ( $I-V$ ) characteristics (at $T=4.2 \mathrm{~K}$ in (a) and at $T=77 \mathrm{~K}$ in (b)) of PbS-EuS(3 nm)-PbS tunneling structure with a single EuS barrier grown on $\mathrm{PbS}$ substrate. The solid lines show the best theoretical fit obtained using the Simmons expression for the tunneling current and taking into account an additional resistor $R_{\mathrm{s}}$ connected in series with the tunnel resistor. The dashed lines illustrates the effect of the resistor $R_{\mathrm{s}}$ on the $I-V$ characteristics (here: $R_{\S}=0$ and all the other parameters are the same as in the calculations shown by the solid lines). The dotted lines present the best fits for the model without the $R_{\text {s }}$ resistor.
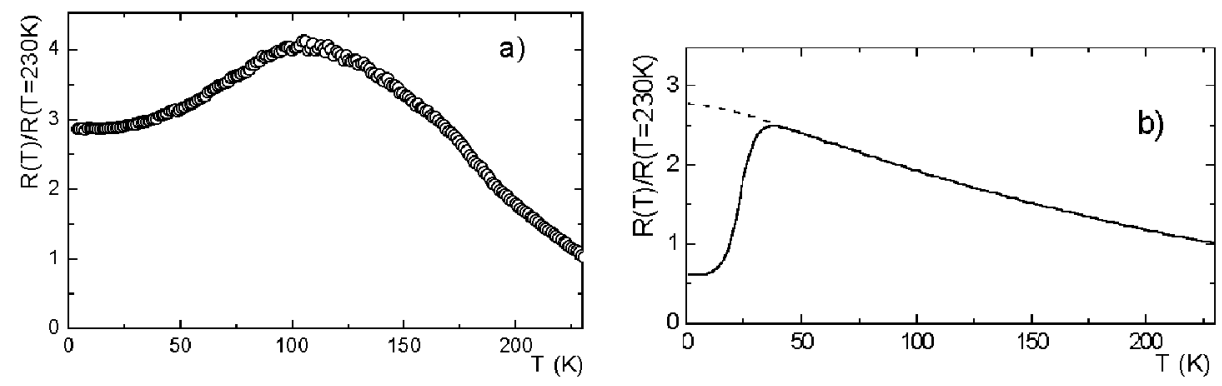

Fig. 3. The temperature dependence of the tunneling resistance of $\operatorname{PbS}(100 \mathrm{~nm})-$ $\operatorname{EuS}(3 \mathrm{~nm})-\mathrm{PbS}(60 \mathrm{~nm})$ single barrier structure grown on PbS (100) substrate (a). Part (b) presents this dependence modeled taking into account the linear decrease (with increasing temperature) of the potential barrier for electrons due both to the increase in the energy gap of PbS and the decrease in the energy gap of EuS. At low temperatures, the expected dominant effect is due to the decrease in the electronic barrier brought about by the exchange splitting present below the ferromagnetic Curie temperature of EuS (solid line). For comparison, the case of "paramagnetic" (no exchange splitting) EuS is also shown (broken line). 
field or applying small field in the plane of the structure. The PbS-EuS multilayer structures exhibited a pronounced non-linearity in the current-voltage $I-V$ characteristics for vertical, i.e., along the growth direction, electron transport. The typical example of the $I-V$ characteristics of the trilayer structure with a single EuS barrier is presented in Fig. 2 at $T=4.2 \mathrm{~K}$ (below the Curie temperature) and $T=77 \mathrm{~K}$ (above the Curie temperature). The results of a model theoretical analysis of this $I-V$ curve (solid and broken lines in Fig. 2) are given in the next section. Figure 3 presents the temperature dependence of the resistance (at low bias of $V=90 \mathrm{meV}$ of the $\mathrm{PbS}-\mathrm{EuS}(4 \mathrm{~nm})-\mathrm{PbS}$ trilayer structure over the temperature range $T=4.2-230 \mathrm{~K}$. The non-monotonic dependence of resistance on temperature with a broad maximum about $T=100 \mathrm{~K}$ observed in most of the structures studied is discussed in the next section.

\section{Discussion and conclusions}

It is expected that the dominant mechanism of the vertical (along the growth direction of the multilayer) electron transport in $\mathrm{PbS}-\mathrm{EuS}-\mathrm{PbS}$ trilayers is due to the electron tunneling from $n$-PbS layers via the spin-dependent EuS ferromagnetic barrier. The experimentally observed strongly non-linear $I-V$ characteristics of the EuS-PbS heterostructures can indeed be modeled using, e.g., the well-known Simmons expression for metallic electron tunneling structures [7]. However, the more detailed analysis of the $I-V$ experimental curves shows that the fully satisfactory theoretical description is obtained if the equivalent electrical circuit of $\mathrm{PbS}-\mathrm{EuS}-\mathrm{PbS}$ trilayer structures contains additional (about $100 \Omega$ ) resistor $R_{\mathrm{s}}$ connected in series with the tunnel resistor (see Fig. 2). This additional resistor is likely to originate, e.g., from the non-perfect ohmic contacts or from $\mathrm{Au} / \mathrm{PbS}$ interface resistance. For comparison, we also show in Fig. 2 the $I-V$ characteristics calculated without this additional resistor. We would like to note that our modeling indicates that the effective electron tunnel barriers found in our structures are of the order of $0.5-0.2 \mathrm{eV}$, i.e., they are about a factor of two smaller than expected based on the photoluminescence experiments and the tunneling studies of EuS-metal hybrid structures. Additionally, we also observed that although, as expected, tunneling barriers at $T=77 \mathrm{~K}$ (above the ferromagnetic transition) are systematically found to be larger than the barriers at $T=4.2 \mathrm{~K}$ (below the Curie temperature), the difference is much smaller than expected. These experimental findings suggest that the EuS electron barriers in EuS-PbS structures studied in this work are rather imperfect. The same conclusion may be reached based on the experimentally found lack of any significant effect of the application of the low in-plane magnetic field on the $I-V$ characteristics. Finally, we mention that in some of PbS-EuS structures studied, we found only weakly non-linear $I-V$ characteristics with rather low resistance and strongly reduced effective barrier height. The vertical current transport in these structures is likely to proceed via pin-hole 
electrical microbridges or other interface defects that electrically short-circuit the EuS barrier.

Figure $3 \mathrm{~b}$ presents the results of our model analysis of the temperature dependence of the resistance of $\mathrm{PbS}-\mathrm{EuS}-\mathrm{PbS}$ structures. Our conclusion is that at high temperatures, $T>100 \mathrm{~K}$, the experimentally observed decrease in the resistance of the structures is likely to originate from the effect of a decrease in the electron tunneling barrier $U$ at the EuS-PbS interface, $U=E_{\mathrm{g}}^{\mathrm{EuS}}-E_{\mathrm{g}}^{\mathrm{PbS}}$, brought about by the simultaneous linear decrease in the energy gap of EuS and the increase in the energy gap of $\mathrm{PbS}$. The model calculations presented in Fig. 3b correctly reproduce both the overall temperature dependence and the magnitude of the effect. At low temperatures (below the Curie temperature) one expects the decrease in the tunneling resistance due to the spintronic effect related to the decrease in the electron barrier $U$ as governed by the exchange splitting of the conduction band of EuS. Although qualitatively correct, our model predicts the maximum on the resistance vs. temperature plot at temperature considerably lower than found experimentally.

In conclusion, we have studied experimentally and developed a simple model of the strongly non-linear $I-V$ characteristics of PbS-EuS-PbS tunneling ferromagnetic trilayer structures. Our analysis indicates that EuS electronic barriers are in these structures considerably reduced as compared to the ones concluded from optical studies. At high temperatures, the experimentally observed linear decrease in the tunnel resistance can be satisfactory described by the electron tunneling mechanism taking into account the temperature dependence of the band structure parameters of the EuS-PbS heterointerface.

\section{Acknowledgments}

This work was supported in Poland by grant PBZ-KBN-044/P03/2001 and within European Community program ICA1-CT-2000-70018 (Centre of Excellence CELDIS).

\section{References}

[1] I.V. Kolesnikov, V.A. Litvinov, A.Yu. Sipatov, A.I. Fedorenko, A.E. Yunovich, Sov. Phys.-JETP 67, 1431 (1988).

[2] A. Mauger, C. Godart, Phys. Rep. 141, 51 (1986).

[3] S. von Molnar, J. Supercond. 16, 1 (2003).

[4] A. Stachow-Wójcik, T. Story, W. Dobrowolski, M. Arciszewska, R.R. Gałązka, M.W. Kreijveld, C.H.W. Swuste, H.J.M. Swagten, W.J.M. de Jonge, A. Twardowski, A.Yu. Sipatov, Phys. Rev. B 60, 15220 (1999).

[5] T. Story, Phys. Status Solidi B 236, 310 (2003).

[6] L. Kowalczyk, J. Sadowski, R.R. Gałązka, A. Stachow-Wójcik, A.Yu. Sipatov, V.A. Smirnov, Acta Phys. Pol. A 94, 357 (1998). 
[7] J.S. Moodera, G. Mathon, J. Magn. Magn. Mater. 200, 248 (1999).

[8] P. LeClair, J.K. Ha, H.J.M. Swagten, J.T. Kohlepp, C.H. van de Vin, W.J.M. de Jonge, Appl. Phys. Lett. 80, 625 (2002).

[9] H. Kępa, J. Kutner-Pielaszek, J. Blinowski, A. Twardowski, C.F. Majkrzak, T. Story, P. Kacman, R.R. Gałązka, K. Ha, H.J.M. Swagten, W.J.M. de Jonge, A.Yu. Sipatov, V.V. Volobuev, T.M. Giebultowicz, Europhys. Lett. 56, 54 (2001).

[10] M. Chernyshova, L. Kowalczyk, A. Szczerbakow, T. Story, C.J.P. Smits, H.J.M. Swagten, C.H.W. Swuste, J.K. Ha, W.J.M. de Jonge, A.Yu. Sipatov, V.V. Volobuev, J. Supercond. 16, 213 (2003).

[11] T. Figielski, A. Morawski, T. Wosiński, S. Wrotek, A. Mąkosa, E. Łusakowska, T. Story, A.Yu. Sipatov, A. Szczerbakow, K. Grasza, J. Wróbel, W. Palosz, J. Supercond. 16, 183 (2003).

[12] M. Chernyshova, E. Eusakowska, V. Domukhovski, K. Grasza, A. Szczerbakow, S. Wrotek, L. Kowalczyk, T. Story, C.J.P. Smits, H.J.M. Swagten, W.J.M. de Jonge, W. Palosz, A.Yu. Sipatov, V.V. Volobuev, Acta Phys. Pol. A 102, 609 (2002).

[13] A. Szczerbakow, Cryst. Growth Des. 1, 183 (2001).

[14] K. Grasza, W. Palosz, Cryst. Res. Technol. 34, 565 (1999). 